
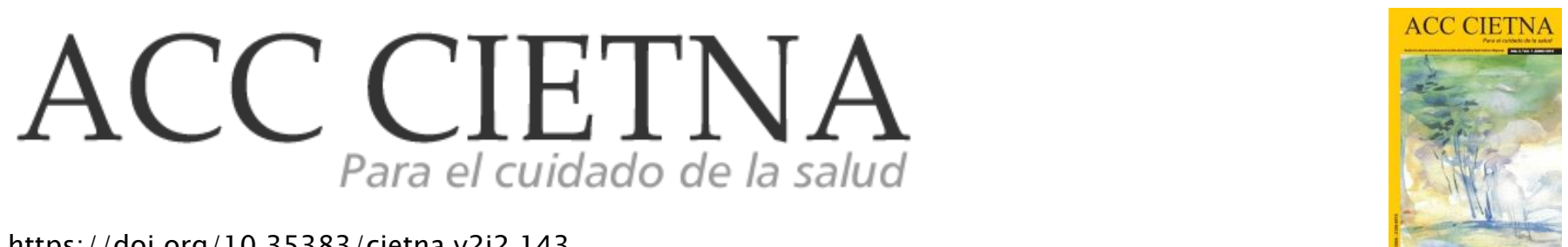

https://doi.org/10.35383/cietna.v2i2.143

\title{
Sentido de vida/muerte de los docentes de la Escuela de Enfermería, que laboran en Unidades Críticas. USAT. Chiclayo, 2014
}

\author{
Torres Malca Dany ${ }^{1}$, Zapata Rueda Aurora²
}

\begin{tabular}{l} 
INFORMACIÓN DEL ARTÍCULO \\
\hline Historia del artículo: \\
Recibido el 23 de julio de 2015 \\
Aceptado el 15 de noviembre de 2015
\end{tabular}

\section{Palabras claves:}

Sentido de vida

Muerte

Fenomenología

Unidad de Cuidados Críticos

\section{RESUMEN}

Esta investigación tuvo como objetivos: describir, analizar y comprender el sentido de vida/muerte de los docentes de la Escuela de Enfermería de la Universidad Católica Santo Toribio de Mogrovejo, que laboran en Unidades Críticas. El referencial teórico estuvo basado en conceptos de sentido de vida de Yepes (1996), Frankl (1991), Castro (2006); de muerte, según Osadolor (2003), Monge (2007), Málishev (2007), y concepto de unidades críticas, según Perdomo (2004). La metodología fue cualitativa con abordaje fenomenológico, postulado por Stein (1998). Los sujetos de estudio fueron 10 enfermeros, docentes de la universidad mencionada; a quienes se les aplicó una entrevista a profundidad. Se obtuvo como resultado 6 proposiciones: Dios creador de la existencia humana con un fin, descubriendo el sentido de vida desde los cuidados de enfermería, descubriendo el sentido de vida con libertad y autorrealización en familia, la muerte hecho inevitable doloroso de aceptar, acompañando hasta el final con oración y fe en Dios y descubriendo el sentido de vida desde el pensamiento de la muerte. Se concluyó que el sentido de vida y muerte del docente se circunscribe a las experiencias que posee en el cuidado a las personas en fase terminal, y más aún les cuesta interiorizar su realidad de finitud; pues más hacen referencia a la muerte del "otro" y ven muy lejana su propia muerte, aun así se sienten identificados por el dolor de la familia y del propio moribundo.

\footnotetext{
1 Licenciado en Enfermería. Enfermero con trabajo independinte, Chiclayo, Perú. Email: dantm17@gmail.com

${ }^{2}$ Licenciada en Enfermería. Docente de la Escuela de Enfermería de la Universidad Católica Santo Toribio de Mogrovejo, Chiclayo, Perú. 
Sense of Life / Death of Teachers from Nursing School of Santo Toribio de Mogrovejo University that works on Critical Care Units. USAT. Chiclayo 2014

\section{ABSTRACT}

Keywords:

Sense of life

Death

Phenomenology

Critical care Unit
The research entitled "Sense of Life / Death of Professors from Nursing School of Santo Toribio de Mogrovejo University who work on Critical Care Units. Chiclayo, 2014", let us to: describe, analyze and understand the sense of life / death of these professors. The theoretical framework was based on the concepts of: Sense of life Yepes (1996), Frankl (1991), Castro (2006) death of Osadolor (2003), Monge (2007), Malyshev (2007) and critical units according Perdomo (2004), The methodology was qualitative with a phenomenological approach, postulated by Stein (1998). The subjects were 10 university professors who work on critical care units; the depth interview was applied to collect data. Results: Six prepositions resume the results of the research: God the Creator of human existence with a goal, discovering the meaning of life from nursing care, discovering the meaning of life with freedom and selfrealization into the family. Death: inevitable painful fact to accept. Accompanying the end of life with prays and faith in God. Discovering the meaning of life through death thought. Finally, the meaning of life and death of the professor is limited to the experiences he has caring people in terminally phase, and it's a struggle for them to internalize the reality of finitude; They make more reference to the death of "others" but they see, their own death far from this time, but they identify themselves with the pain of the family and patients.

\section{Introducción}

Desde la creación del hombre, la relación que establece con otros seres de su misma especie, le imprime el carácter de ser social por naturaleza. Se dice que es un ser social de por sí, ya que habita en un entorno donde no está solo, sino donde se relaciona con otras personas, cada una de ellas con costumbres, intereses e ideas propias, con pensamientos llenos de sueños, proyectos, metas que desean cumplir durante su vida, lo que le permite desarrollarse como ser humano útil para su sociedad'.

Pero muchas veces dentro de esa sociedad la persona en su interior no sabe cuál es el motivo de su xistencia, sin apartarse de la sociedad actual, Viktor Frankl explica que la neurosis que predomina en la sociedad es la neurosis noógena caracterizada por la falta de sentido de la vida que padecen las personas y que él denomina como vacío existencial. Este vacío existencial constituye una "pérdida del sentimiento de que la vida es significativa", las personas presentan "el sentimiento de que sus vidas carecen total $y$ definitivamente de un sentido. Se ven acosados por la experiencia de su vaciedad íntima, del desierto que albergan dentro de sí". En definitiva, quien padece un vacío existencial considera que "la vida no tiene sentido" y que "no vale la pena vivirla"2. 
Las personas durante su paso por la diferentes etapas de vida experimentan situaciones de conflicto y frustración, que les lleva aparentemente a no encontrarle sentido a su vida, planteándose interrogantes sobre su existencia como ¿tiene sentido mi vida en este mundo?, ¿por qué existo?, ¿para quién vivo?, ¿es necesario estar vivo para buscar la felicidad?, etc. Sin embargo, las respuestas a estas preguntas no siempre son positivas y esto puede evidenciarse en el alto número de suicidios que se registran en nuestro país. Tal y como lo demuestra el Instituto de Salud Mental Honorio Delgado - Hideyo Noguchi, que en el 2012 registró 305 casos de suicidios, y en el 2013,334; esto significa que el nivel de suicidios en el país ha ido en aumento, aproximadamente un $9 \%$, principalmente suceden por problemas sentimentales entre parejas, crisis económicas, estados depresivos y enfermedades físicas. En lo que va del año 2014 esa cifra ya ha sido superada ${ }^{3}$.

Frente a esta realidad, es el personal de enfermería quien desarrolla un rol importante para cuidar de la salud de las personas, y no solo de una salud física; sino que el enfermero está llamado a cuidar la salud integral de las personas y esto pasa por contribuir a que las personas descubran un sentido a su existencia.

Durante el cuidado de la salud de las personas, el enfermero suele encontrarse con la realidad del morir, pudiendo asumir su cuidado como una lucha contra la muerte. $Y$ no sólo con la muerte misma sino con todo lo que ella desencadena y le antecede, como es el dolor, el sufrimiento, la incomprensión, entre otros. Pues la muerte lejos de ser contraria a la vida, forma parte de esta. Francesc Torrealba refiere que "si la muerte es inexorable y además igual para todos, entonces es preciso asumir plenamente en la vida humana este acontecimiento tan cotidiano y por otro lado tan excepcional" 4.

Cualquier escenario es propicio para la muerte, pero son las salas de cuidado críticos las que inclinan a las experiencias de vivir y morir, de sufrimiento y dolor, de esperanza y desesperanza y son estas salas en las que el profesional de enfermería se plantea interrogantes existenciales sobre el sentido del sufrimiento humano, la vida y la muerte, pues se encuentra entre esta dualidad cuando cuida, y quizás se cuestione: ¿es un fracaso profesional el morir de un paciente?, ¿realmente se lucha contra la muerte? Ante estas interrogantes el enfermero puede responder muchas veces con una actitud de aparente indiferencia, o de simpatía, que puede verse reflejada en la rutina diaria de trabajo.

El investigador durante su formación ha realizado prácticas hospitalarias en diferentes servicios de unidades críticas, que le han permitido observar que el profesional de enfermería, durante el cuidado hacia una persona en el proceso de morir, parece ser indiferente, y sentir rechazo o temor. Otros se sienten limitados porque la comunicación disminuye, entonces nos distanciamos de la persona, pues aparentemente "no hay nada más por hacer".

Asimismo, existe una fuerte tendencia a evitar el dolor de la persona que agoniza, por lo que los cuidados al final de la vida se atomizan a eso, la evitación del dolor, quedando aún pendiente esa relación fenoménica entre el cuidador y la persona que muere. ¿Cómo se altera la vida de una enfermera cuando sus pacientes mueren?, ¿qué significado tiene para el personal de enfermería, el dolor de las personas que se encuentran en cuidados críticos, cuya vida se mantiene por procedimientos invasivos que a pesar de mantenerle con vida no dejan de ser lesivos para su integridad corporal y emocional?

Las consideraciones anteriores y el interés por el investigador permitieron plantear el siguiente problema de investigación: ¿cuál es el sentido de $\mathrm{vida} /$ muerte de los docentes de la escuela de enfermería que laboran en unidades críticas. USAT. Chiclayo, 2014? Se tuvo como objeto de estudio el entido de vida/muerte del docente de la escuela de enfermería que labora en unidades críticas. Los 
objetivos que guiaron el presente estudio describir, analizar y comprender el sentido de la vida/muerte de los docentes de la escuela de enfermería que laboran en unidades críticas.

La presente investigación se justificó dado que existen investigaciones que demuestran que las enfermeras viven el proceso de muerte con miedo a la pérdida, manifestado con dolor, impotencia y tristeza. El dolor y la tristeza se relacionan con el tiempo de convivencia y lo que le significa el paciente. La impotencia de no poder hacer más por el paciente y finalmente no saber cómo apoyar a la familia $^{5}$. Sin duda alguna, esta forma de vivir el cuidado de la persona en fase terminal corresponde al sentido que tiene para ellas la vida y la muerte, pues como afirma Frankl quien le ha encontrado un sentido a la muerte le ha encontrado un sentido a la vida? .

Por otro lado, las enfermeras de las unidades de cuidados críticos se enfrentan día a día con preguntas existenciales de las personas a su cuidado, como ¿por qué Dios permite que sufra? o ¿qué sentido tiene el sufrimiento? Preguntas que requieren respuestas que desbordan el plano meramente tecnológico y biológico, pues reclaman una interiorización de las enfermeras en su sentido de vida y sentido de muerte. Interiorización que solo es posible cuando se posee una base antropológica que sustente el significado de persona humana a quien cuida.

Además, esta investigación servirá para reconocer la necesidad de fortalecer el conocimiento antropológico del enfermero docente que trabaja en las unidades críticas, es decir, el conocimiento del mundo de forma integral, con sus errores, límites y defectos, así como permitir nuevas investigaciones, capacitaciones, programas, $y$ mejora de los diseños curriculares, que desde la formación universitaria debe realizarse para poder comprender esa finitud de la vida, desde el nacimiento hasta su término mortal, que de acuerdo al significado de su propia existencia encontrará el fin último.

\section{Metodología}

La investigación fue cualitativa, con abordaje fenomenológico. Este enfoque fenomenológico explora la "estructura de consciencia" en "experiencias humanas" ${ }^{6}$. Las etapas del enfoque fenomenológico fueron: descripción, reducción y comprensión del fenómeno ${ }^{7}$. Los sujetos de estudio fueron 10 docentes de la escuela de enfermería USAT que laboran en las unidades críticas. La recolección de datos fue a través de la entrevista a profundidad, el número de participantes se delimitó aplicando los principios de saturación y redundancia. Los criterios de inclusión fueron: docentes enfermeros que laboren dentro de las unidades críticas y que desearan participar en la investigación. Se asignaron seudónimos a fin de respetar su identidad. Estos seudónimos obedecían a apelativos escogidos por los propios participantes. El escenario lo constituyeron las unidades de cuidados críticos. Se realizó un análisis de datos siguiendo los pasos de la trayectoria fenomenológica y utilizando el análisis ideográfico y nomotético.

El análisis ideográfico consiste en la transformación de las proposiciones del sujeto, basado en la utilización de ideogramas representando ideas por medio de la escritura, a través de la lectura y relectura de los ideogramas, para comprender el fenómeno develado. El análisis nomotético, es elaborado después de haber concluidos con los análisis individuales de los discursos, así se desarrolló un análisis de lo general, que es la búsqueda de las generalidades del fenómeno interrogado6. Se puntualizó la esencia final del fenómeno, a través de la obtención de resultados de la comprensión de convergencias y divergencias presentes en los discursos.

Del mismo modo, se emplearon los momentos sustentados por Martins y Bicudo7: sentido del todo, discriminación de las unidades de significado, transformación de expresiones del 
sujeto en una lengua psicológica y síntesis de unidades de significados transformadas a proposiciones 6 .

Durante toda la investigación se usaron los principios de la bioética personalista: el valor de la vida humana, principio de libertad $y$ responsabilidad, principio de totalidad, y principio de socialización y subsidiariedad ${ }^{8}$. Se aplicaron los criterios de cientificidad: credibilidad y auditabilidad ${ }^{9}$.

\section{Resultados, análisis y discusión}

Luego de haber obtenido los datos necesarios para dar respuesta al objetivo planteado, la información fue transcrita, ordenada y analizada, obteniendo las siguientes proposiciones:

I. Dios creador de la existencia humana con un fin.

II. Descubriendo el sentido de vida desde los cuidados de enfermería.

III. Descubriendo el sentido de vida con libertad y autorrealización en familia.

IV. La muerte, hecho inevitable doloroso de aceptar.

V. Acompañando hasta el final con oración y fe en Dios.

VI. Descubriendo el sentido de vida desde el pensamiento de la muerte.

\section{Dios creador de la existencia humana con un fin}

Los docentes enfermeros que laboran en unidades críticas al reflexionar profundamente sobre su ser y su propio yo, concluyeron que Dios, a través de sus padres, les ha otorgado la vida, que se hace fecunda durante su desarrollo personal y profesional, aspecto que se evidencia en el siguiente discurso:

"Yo existo, porque no vine por venir, sino que Dios hizo que se unieran mis padres y me procrearan, además me otorgó un objetivo de vida, el cual fue ser enfermera para ayudar a las personas enfermas".

Sol
La vida se presenta como un fenómeno natural cuyo origen, evolución y fin se desenvuelve en el planeta que habitamos ${ }^{10}$. El considerar de esa forma la vida, le permite al enfermero de UCl optar por una actitud concreta sobre ella, conocer su propio ser y su propio yo, debido a que reflexiona sobre sí mismo, percibe la moralidad de las propias acciones, trasciende con su conocimiento y su voluntad, pero sobre todo con su libertad, el resto del mundo. Stein menciona que el ser de la persona se da desde la perspectiva de la experiencia del propio ser y del propio yo ${ }^{11}$.

Darse cuenta del propio ser, implica al enfermero llevar consigo un conjunto de vivencias, experiencias, luchas, éxitos, fracasos dentro de un espacio y en un tiempo determinado, en este caso la sala de UCI se convierte en lugar especial, donde comparten momentos únicos, que les lleva a tener un sentido concreto de la vida e importancia para seguir estando vivo en este mundo.

La aceptación de Dios como creador único del ser humano se da por la dimensión religiosa, porque en la persona existe el deseo natural de verdad, de bien y de felicidad, que son manifestaciones naturales de ver a Dios, estas aspiraciones constituyen el núcleo más profundo de la vida espiritual y dignidad de la persona. Su presencia en lo más profundo del corazón muestra la existencia de su Creador ${ }^{12}$.

El fin último o bien perfecto de la vida humana, significa objeto último de deseo, fin o bien global en el que los deseos quedan satisfechos, que no deja nada importante fuera de sí y que no es referible a ningún fin ulterior o, lo que es igual, es querido por sí mismo y no en orden a otra cosa13.

Los enfermeros al comprender que su ser tiene significado cuando tienen algo por realizar, ya sea dentro de las salas de $\mathrm{UCl}$ o donde se encuentren, también forma parte de su pensamiento, que su ser es motivado por un ser superior, es decir Dios, quien les otorga la vida a su imagen y semejanza, 
además es quien les da un fin, los objetivos y metas para poder ir desarrollándose como persona y profesional. De allí que se origine la siguiente preposición:

\section{Descubriendo el sentido de vida desde los cuidados de enfermería}

Los enfermeros docentes al estar en contacto con el dolor $y$ el sufrimiento de las personas hospitalizadas han dejado huella en lo profundo del ser de la enfermera, estos sucesos les condujeron a reflexionar y tomar decisiones importantes en sus vidas, de tal manera que debieran cumplir adecuadamente sus roles de madre, mujer y enfermera, como refieren el siguiente discurso:

"En mi vida, trato de dar ese sentido humano de la profesión, dando cariño, amor, a los demás, y enseñando a mi familia a encaminarse hacia un objetivo, viviendo en paz y armonía, como enfermera, aliviando el dolor físico y psicológico que pasa el paciente y su familia; ya que algunos manifiestan "me voy a morir, tengo miedo, me va pasar algo" entonces yo estoy ahí, quizás para tranquilizar, dando apoyo emocional, a través del diálogo, expresándoles que vamos a superarlo junto con los médicos, la ciencia y la ayuda de Dios..." Sol

Stein menciona que todo ser humano posee un ethos vocacional, de tal forma que caracteriza al ser de otras vocaciones, es decir, un estado anímico o totalidad de hábitos que en la vida profesional se presentan como principio intrínsecamente configurador ${ }^{14}$.

Ese ethos profesional está presente en las enfermeras de $\mathrm{UCl}$, porque lo han desarrollado a través de su formación o le han ido descubriendo en el cuidar a las personas en la unidad de cuidados intensivos, $y$ esto les ha permitido a través de las diferentes experiencias en esta unidad, brindar un cuidado destacado, generoso y noble, que permite mejorar la calidad de vida de éstas personas.

Por ello, todo cuidado ofrecido en los diferentes escenarios de servicio está dirigido a satisfacer las necesidades del individuo ya sea que el ser humano esté en salud o enfermedad. Es por esto, que el enfermero aplica diferentes disciplinas en el cuidado, visualizando al individuo como un ser integral, biológico, psicológico, sociológico, espiritual y un ser holístico ${ }^{15}$.

Las enfermeras, las cuales toman una actitud concreta referente a su vida, asumen que esta tiene un inicio y un final, guardando la esperanza de alcanzar su realización existencial para lo cual se esmeran en su desarrollo personal y profesional, a fin de sentir satisfacción de que están cumpliendo con el fin por el que fueron creadas o por el que se hicieron enfermeras y que forma parte de su sentido de vida.

Polo refiere que el que vive la esperanza afirma que estamos en un mundo mejorable, y por eso no se instala en el presente, sino que comprende el trayecto que conduce a una meta ${ }^{15}$. De allí, que las enfermeras sean perseverantes en el cuidado a la persona y cuiden con desvelo, generosidad y sean capaces de continuar con su labor a pesar del sufrimiento, el dolor o la muerte que son experiencias con las que convive. Esta distinción en el cuidar se evidencia en la siguiente preposición:

\section{Descubriendo el sentido de vida con libertad y autorrealización en familia}

Los enfermeros de áreas críticas argumentan que su vida tiene sentido cuando se sienten en el propio seno familiar, actuando con autonomía sobre sus decisiones $y$ acciones personales o profesionales, tal y como se muestra a continuación:

"Mi vida trato de llevarla por una dirección correcta, a través del buen uso de mi libertad, usar bien mi libertad se refiere a que yo decido y asumo 
las consecuencias de mi actos, donde me encuentre.

Además mi vida la disfruto de muchas maneras, ya sea viajando, reuniéndome con amigos $o$ conviviendo con la familia para sentirme satisfecha de seguir viviendo..."Chinita

La libertad se pone interesante desde el momento en que asume tareas importantes $y$ comprometidas.

Basta pensar en qué es la vida profesional para darse cuenta de que ser libre exige llenar la vida de contenido, tener un lugar qué ocupar en la sociedad 17 .

Las enfermeras manifiestan ser libres al asumir con gran responsabilidad todas sus acciones $y$ decisiones dentro de su entorno personal, familiar, social o laboral. Es decir, tienen claro los objetivos y metas que deben cumplir, lo que les permite estar encaminadas a su realización en este mundo. Para ello, muestran gran dedicación, compromiso, esfuerzo y amor en cada cuidado hacia el prójimo.

Las enfermeras del grupo de estudio han descubierto su sentido de vida, por medio de su propio conocimiento sobre su ser, al descubrir a Dios como creador. A todas estas vías del sentido, se le agrega la familia, tal y como se muestra a continuación:

"Mi vida tiene sentido cuando oriento a mis hijos para que sean unas personas de bien, además cuando en mi familia hay amor, hay buena comprensión, responsabilidad, lealtad y fidelidad."

\section{Adriana}

La familia constituye la célula básica de la sociedad, o sea su institución más simple, basada en los lazos de parentesco conyugal y consanguíneo, que se establecen por la vía del matrimonio y la procreación de los hijos ${ }^{18}$.

Las enfermeras de $\mathrm{UCI}$, al enfrentarse día a día a situaciones críticas cómo perder a un ser querido o ver sufrir a los familiares cuando un niño, joven o adulto se hallan en riesgo de morir, reflexionan y comprenden que lo más valioso para dar sentido a su vida es su propia familia, debido que en ella, se muestran grandes sentimientos de amor, cariño, afecto, para poder crecer y desarrollarse dentro de la sociedad. Todas estas expresiones de afectividad, les permite a los enfermeros tener una vida plena con gran seguridad de sí misma para efectuar sus cuidados antes, durante y después de situaciones inesperadas o críticas.

Entre estas situaciones, está la presencia de morir que resulta por ser un hecho doloroso tal como se describe en la siguiente preposición:

\section{La muerte, hecho inevitable doloroso de aceptar}

Así como el hombre reflexiona sobre su vida, también lo hace sobre su muerte, su origen, sus causas, su significado y sus consecuencias. Las enfermeras de UCI no son ajenas al significado de esta realidad; en su trayecto de vida, han procurado entenderla de muchas maneras posibles, ya sea como fin de la vida, paso de esta vida a la vida eterna, algo natural, encuentro con Dios, todo esto motivado por la pérdida de un ser querido, que desencadenó en ellas sentimientos y emociones de tristeza, pena, frustración, etc., o también al ver morir a sus pacientes, tal y como se muestra a continuación:

"La muerte es la ausencia de las funciones vitales de una persona, a veces es lamentable estar presente cuando alguien deja de existir, porque veo y siento ese sufrimiento de las personas, sobre todo si es un persona pequeña con poco tiempo en el mundo, me da nostalgia, pena, al presenciar el dolor de los padres, pero si es una persona que ha tenido un rol y ha gozado buen tiempo de su vida, me siento más tranquila, a pesar de tener años de experiencia no puedo superar la pérdida y no tengo las palabras adecuada para poder comunicar a los familiares..." Clavel

La muerte se considera frecuentemente como el acto final en el proceso vital y por lo tanto, forma 
parte de esta. La enfermería, como profesión implica ayudar a personas sanas o enfermas, en la realización de aquellas actividades que contribuyan a la salud, a su recuperación o a una muerte tranquila; esta última puede ser especialmente dolorosa, ya que quizás la situación implique sentimientos de culpa, de haber fallado, tristeza, impotencia, frustración, incluso cuando se hizo todo lo posible. A menudo no sabemos qué decir y nos sentimos incapaces de ofrecer palabras de consuelo19.

La tristeza ante la muerte se da porque la razón acepta que la muerte es ineludible, pero el corazón no acepta que lo sea. La pena se da frente a la pérdida física de la persona. La impotencia, sucede al tener la incapacidad para poder realizar algo, frente a lo ineludible. La frustración, se da frente a la ira y la decepción, que surge de la percepción de la resistencia al cumplimiento de la voluntad individual. La causa de la frustración puede ser interna o externa ${ }^{19}$.

Todos esos sentimientos y emociones relacionados con la muerte, impulsan a los profesionales de enfermería a prepararse para brindar los cuidados al paciente y a la familia, con conocimientos científicos, éticos, humanísticos, conscientes de que en la medida de su instrucción podrán desempeñar más eficazmente las tareas del cuidado, a la vez que adquieren las herramientas para afrontar o reducir su propio miedo, temor o ansiedad ante la muerte de un paciente o de alguien cercano. Una de las formas que los enfermeros emplean para hacer más llevadero este proceso de morir es la oración y su fe en Dios, tal como se evidencia en la siguiente preposición:

\section{Acompañando hasta el final con oración y fe en Dios}

Los enfermeros de $\mathrm{UCl}$ viven y experimentan la muerte de manera continua, permitiéndoles darse cuenta que su vida tiene finitud, que por más ciencia y tecnología para cuidar de las personas es necesario acudir a un ser superior, para entender la vida y la muerte. Este medio lo constituye la oración, como fuente de fortaleza para cuidar y ayudar a las personas a que no sufran en sus últimos momentos, tal como se evidencia a continuación:

"El medio que me ayuda a convivir con la muerte es la oración, porque cuando muere alguien, yo realizo una oración internamente, para acercarlo a Dios, o a veces hago que en los últimos momentos, la familia lo (la) acompañe hasta que muera" Jacofa

El hombre que sabe orar vive en contacto con Dios, sabe el valor de los beneficios que de su Padre recibe y a la vez comprende el sentido o la finalidad de las pruebas por las que atraviesa. Gracias a ella se logra la paz interna, se adquiere sabiduría, se comprende lo profundo, ilumina nuestra mente y fortalece nuestro espíritu, sin ella no tendríamos la fuerza necesaria para afrontar cada proceso de duelo, que se nos presenta a lo largo de la vida 20 .

Las enfermeras al estar en contacto con el dolor, el sufrimiento y la muerte de sus pacientes dentro de las salas críticas, sienten que necesitan acudir a un ser superior por medio de la oración, para tener fortaleza, seguridad, serenidad y confianza para ayudar en los momentos más difíciles que el paciente y su familia se hallan pasando, esta forma de vivir, les permite comprender que la oración es la fuerza espiritual para poder afrontar y superar cada proceso de duelo que la vida misma nos presenta.

Escrivá menciona que el ser enfermera es una vocación particular de ser cristiana. Pero para que esa vocación se perfeccione es preciso tener conocimientos científicos y delicadeza muy grande con la atención de los pacientes ${ }^{21}$.

Las enfermeras de $\mathrm{UCl}$ al realizar una autorreflexión y utilizar la oración como medio de convivencia con la muerte, hace que su vida tenga una dirección cristiana, con mucha sensibilidad y 
humanismo, a fin de lograr el descanso eterno y el acercamiento a Dios.

Quienes desean acompañar a personas en duelo han de ser conscientes de la importancia, dureza y el influjo del duelo sobre la vida entera, han de ser conscientes de su naturaleza, su proceso, su función y su diversidad. En otras palabras, las enfermeras de $\mathrm{UCl}$ deben mostrar todo ese conocimiento del duelo, al tener una actitud solidaria, de ayuda al paciente y el afrontamiento de pérdida con la familia ${ }^{22}$. Esto le permitirá al enfermero darle sentido a la vida a pesar de ser la muerte la vía para encontrarlo, como veremos a continuación:

\section{Descubriendo el sentido de vida desde el pensamiento de la muerte}

La gran mayoría de las enfermeras de UCI descubrieron su sentido de vida al pensar en su propia muerte, es decir, hicieron una reflexión y un recorrido interno de todo el trayecto de su vida, tal y como lo refiere el siguiente discurso:

"Pensando en la muerte, pienso que mi vida es de importancia, porque cada cosa que realizo, la disfruto, sea con mi familia o en mi trabajo, como enfermera ayudo a que mis pacientes mueran dignamente, a través del diálogo, del apoyo emocional, viendo si están con su ventilador mecánico conectados adecuadamente, y que en el momento indicado que tenga que irse se vaya, ni adelantándole ni atrasándole, cuando tenga que irse que se vaya sin dolor, tranquilo. Eso indica que he gozado mi vida como yo lo he planificado, y que he cumplido con mis objetivos, aceptando la muerte, ya sea por enfermedad o por accidente..." Sol

Dar un sentido a la vida o sucesivos sentidos, a lo largo del tiempo equivale a darse a sí mismo un segundo nacimiento, es la conciencia de lo que deseamos y podemos ser. En los enfermeros de $\mathrm{UCl}$, el trabajo y los actos concretos, personales y profesionales permiten darle significado a su propia vida 23

Los actos concretos se refieren a llevar una vida, con optimismo, alegría, amor, entusiasmo, etc. En lo personal, tratan de disfrutar, gozar toda esa plenitud de vida, a través de la convivencia en familia, cumpliendo sus roles de hijas, madres y esposas. En lo profesional o laboral, buscan dar todo ese aspecto humano durante su turno de trabajo, cumpliendo siempre a cabalidad todas sus funciones y respetando la dignidad de sus pacientes. En otras palabras, el trabajo tiene sentido en la medida en que es una posibilidad de realización concreta de valores creativos. Es lo que le doy al mundo y a la comunidad y eso habla de mí24.

Los enfermeros de $\mathrm{UCl}$ al vivenciar la muerte de otra persona reflexionan su propia muerte y sobre cómo les gustaría morir, entender esta forma la vida, significa llevar una vida correcta, respetando los valores, cumpliendo las metas y preparándose para cuando la muerte llegue. El sufrimiento de otra persona nos ayuda a dar un sentido a la vida, debido que nos muestra a profundidad quiénes somos y ser más compasivos con el sufrimiento de otras personas, en definitiva, el sufrimiento nos ayuda a madurar como persona y profesional, asimismo nos aleja de la vida superficial con la que se vive la vida en general ${ }^{25}$.

La muerte y el bien morir suelen ser conceptuados como un momento de reflexión, que nos permite reconciliarnos con nosotros mismos, y con todos con quienes hemos compartido nuestra vida, dando y recibiendo gracias, perdonando y sintiéndonos perdonados ${ }^{26}$.

Los enfermeros de $\mathrm{UCl}$, al ayudar al buen morir de sus pacientes comprenden la muerte como el espejo en el cual su vida, está vinculada con el diálogo de su corazón y su espíritu, es decir el corazón ofrece la energía para la acción, y el espíritu ofrece un viaje hacia el crecimiento. Entender esto, significa que la vida misma no es 
más que un periodo pequeño de nuestra existencia 26 .

Cabe señalar que en la vida misma encontramos el significado de la existencia y en la muerte encontramos el significado de la vida, el convencimiento de nuestra muerte nos impulsa a trabajar, a hacer, a producir, sin posponer inútilmente nuestro destino. La presencia de la muerte nos pone frente a nuestra responsabilidad, que es la de hacer de la vida el sentido mismo de la existencia 26 .

\section{Conclusiones}

Se obtuvieron las siguientes consideraciones finales:

En primer lugar, las enfermeras docentes de $\mathrm{UCl}$ al cuestionarse ¿por qué existen? ¿qué sentido tiene su vida?, reflexionan profundamente y llegan a comprender que su vida es otorgada por un ser superior, manifestada por la unión entre el hombre y la mujer, es decir, nuestros padres como partícipes de la vida humana. Esta forma de reconocer y comprender la existencia les ayuda a darse cuenta que no existen por existir, sino que alguien superior a su ser, les ha dado un fin $u$ objetivo que deben cumplir en este mundo. En este caso al ser enfermera.

Así mismo, para las enfermeras docentes el estar en contacto a diario con el dolor, el sufrimiento y con personas al borde de la vida y la muerte, les permite reconocer que su propia profesión también contribuye a darle sentido a su vida, es decir, toda esa vocación, esfuerzo y dedicación con el prójimo por ayudarlo a recuperar su salud dentro de las unidades de cuidados intensivos, les impulsa a cumplir con los objetivos que Dios les ha otorgado en este mundo, de esta manera todas sus acciones personales y profesionales son direccionadas a mejorar la calidad de vida de las personas, con la finalidad de sentirse satisfechas por haber dejado un cambio en la vida de los demás. Todo esto permite a la enfermera tomar una actitud concreta sobre la vida e ir alcanzando su realización existencial, es decir, que en la medida de sus propios esfuerzos tratan de cumplir con todas sus metas u objetivos, para así darle sentido, al "estar vivo" en este mundo.

Las enfermeras docentes, se relacionan a cada instante con distintas personas, dentro de esa interrelación se preguntan ¿qué es lo que hace que mi vida tenga un sentido?, la respuesta está, en la medida que el propio ser o nuestro yo encuentra, cuál es la fuerza que lo impulsa para seguir existiendo. En la gran mayoría de los discursos estudiados, al reconocer que tienen esa capacidad de ser libres para actuar y tomar las decisiones correctas sobre la propia vida, también les ayuda a entender que la familia se convierte en el eje principal del sentido de la vida personal y profesional, debido que cada momento disfrutado con ellos, le permite ser felices y estar agradecidas por todo lo que Dios les has dado.

Sin embargo, hay momentos difíciles e inevitables en la vida que nos deja muchas veces sin la debida explicación verdaderamente convincente del porqué sucedió, este hecho inevitable lo constituye la muerte, ya sea de un familiar o de un paciente, lo cierto es que es muy difícil poder aceptarla y comprenderla, a tal punto que se halle un sentido, muchas de las enfermeras de $\mathrm{UCl}$ ven a la muerte como algo natural, un paso de esta vida a la vida eterna, algo finito, que sucederá en cualquier momento, la forma en la cual sienten que están preparadas y no se dejan sorprender por ella, es entender que forma parte de la vida misma, que todos esos sentimientos y emociones de tristeza, pena, dolor, impotencia, frustración, que desencadena cuando alguien muere, les impulsa a tomar una actitud concreta y convertirlos en fortaleza, valentía, seguridad para afrontar, ayudar, superar y mejorar sus cuidados durante los últimos momentos de la vida humana.

A pesar de tener el conocimiento sobre la vida y la muerte, las enfermeras docentes sienten la necesidad de acudir a un ser superior por medio 
de la oración, para poder ayudarse a sí misma como a los demás, a afrontar la muerte como un hecho inevitable y darle un sentido, llevándolas a entender que la oración dentro de las salas críticas como fuera de ellas, es fuente espiritual, que les da fortaleza, seguridad y confianza para mejorar sus cuidados a sus pacientes terminales como a sus familiares durante el proceso de duelo.

Por último, el gran sentido de la vida, para la mayoría de las enfermeras que laboran en unidades críticas, es comprendido en la medida que es reflexionado desde su propio pensamiento de muerte, es decir, su ser les impulsa a realizar un resumen de todo aquello que han vivido y están viviendo a fin de procurar encontrarle una satisfacción a su propia existencia.

En la medida que trata de hallarlo, reconocen que su vida es un don maravilloso que Dios les ha otorgado, que deben gozarlo y disfrutarlo al máximo, ya sea en la vida personal, familiar o laboral, lo importante es haber cumplido con el objetivo o meta trazada a lo largo de la existencia, siempre buscando transcender o dejado cambios en la vida de los demás.

\section{Bibliografía}

1. Yanellys M. El Hombre como ser social [sitio web]. Economía Social, [acceso 30 Marzo 2014] disponible en: http://es.slideshare. net/yanellys 17012414 /ensayo-del-hombrecomo-ser-social

2. Frankl V. El Hombre en busca de sentido. Duodécima edición. Editorial Herder. Barcelona.1991.

3. El Comercio. Casos de suicidio en el país aumentaron en 9\% durante el 2013". [Diario en internet] sábado 04 de enero 2014. [Acceso 30 Marzo 2014]. Disponible en: http://elcomercio.pe/lima/sucesos/casossuicidiopais-aumentaron-durante-2013-noticia1682248
4. Torrealba F. Antropología del cuidador. Editorial: Instituto Borja de Bioética. Barcelona (España). 1998

5. Vargas E, Pacheco A, y otros. Percepción de enfermería en torno a la muerte de pacientes en etapa terminal" [revista en internet] Revista de enfermería del Instituto Mexicano del Seguro Social 2008; Volumen 16(1): 3744, México. 2008. [acceso 20 Abril 2013] Disponible en: http://revistaenfermeria.imss.gob.mx/

6. Martínez M. Ciencia y arte en la metodología cualitativa". México. Editorial Trillas. 2004.

7. Martins J, Bicudo M. Pesquiza cualitativa en Psicología. Fundamentos y recursos básicos. Sao Pablo - Brasil. Ed. Morales Lda. 1989.

8. Delgado J, Gutiérrez J. Métodos y técnicas Cualitativas de Investigación en Ciencias Sociales. España. Ed. Síntesis. 1995.

9. Serrano J. Principios de Bioética. [Sede web] Universidad Complutense de Madrid. Madrid (España) noviembre 2003. [Acceso 15 Julio 2013]. Disponible en http://www.bioeticaweb.com/content/view/69/4 $0 /$

10. Osadolor I. Logoterapia para mejorar el sentido de vida en pacientes con ansiedad ante la muerte, [tesis doctoral] Universidad Iberoamericana Santa Fe, México. 2005. [acceso 29 Abril 2013]. Disponible en: http://www.bib.uia.mx/tesis/pdf/014538/01453 8.pdf

11. Esparza M. El Pensamiento de Edith Stein. Ediciones Universidad de Navarra S.A. Pamplona (España). 1998.

12. Catecismo de la Iglesia Católica. La Profesión de la Fe, Primera Sección, "Creo - Creemos", Capítulo primero "El hombre es capaz de Dios", 
Deseo de Dios, [online] [acceso 11 Agosto 2013]. Disponible en:

http:/ / www.vatican.va/archive/catechism_sp/pls 1cl_sp.html\#l. El deseo de Dios

13. Rodríguez L. Ética General. 5 taed. Pamplona: Eunsa. España, 2004.

14. Stein E. La Mujer, Según la Naturaleza y la Gracia. $1^{\text {ra }}$ ed. Palabras, Madrid, 1998.

15. Collado R. Cuidado espiritual, labor del profesional de enfermería. Universidad Interamericana de Puerto Rico - Re-cinto de Ponce, Puerto Rico, 2010. [online] [acceso 25 Agosto 2013] Disponible en: http://cremc.ponce.inter.edu/360/ revista360/ ciencia/ Cuidado\%20espiritual\%20enfermeria.pdf

16. Polo L. La esperanza [online] Universidad de Navarra. Pamplona. [acceso 13 Julio 2014]. Disponible en: http://dspace.unav.es/dspace/bitstream/10171/ 13309/1/ST_XXX-1_06.pdf

17. Yepes R. Fundamentos de Antropología. Un ideal de la Excelencia Humana". Sexta Edición. Navarra -España. Editorial EUNSA. 1996.

18. Rodríguez $\mathrm{N}$. Un acercamiento a la familia desde una perspectiva sociológica en contribuciones a las ciencias sociales, Mayo 2012. [online] [acceso 07 Abril 2014].Disponible en: http://www.eumed.net/rev/cccss/20/

19. Vásquez C. Significado de la experiencia de dolor para la enfermera ante la muerte de neonatos. [tesis de maestría] Universidad nacional de Colombia Facultad de Enfermería, Programa de Maestría en Enfermería. Bogotá. D.C. 2012, [acceso 18 Marzo 2014]. disponible en: http:/ / www.bdigital.unal.edu.co/7002/1/539512 .2012.pdf

20. 144000 NET. [sitio web]La Oración Espiritual. [online] [acceso 02 Abril 2014] Disponible en: http:/ / es.144000.net/articulos/la_oracion_espirit ual_2.htm

21. Herranz G. San Josemaría habla a médicos y enfermeras. [sitio web] [Acceso 04 Abril 2014] Disponible en: http://www.es.josemariaescriva.info/articulo/sanj osemaria-habla-a-medicos-y-enfermeras

22. Pérez M. La oración fundamento de la vida cristiana [sitio web] [acceso 04 Abril 2014] Disponible en: http: / / loquecreemos.wordpress.com/laoracionalimento-para-el-espiritu/

23. Logoterapia.com. [sitio web] Vida y trabajo de Viktor e. Frankl. 1945-1947. Una nueva vida para reconvertirse en hombre. Sociedad Mexicana de Análisis Existencial y Logoterapia S.C. (SMAEL) Centro de formación en psicoterapia orientada al sentido. [online] [acceso 15 Abril 2014] Disponible en: http://www.logoterapia.com.mx/ logoterapia/vida-trabajo-viktor-e-frankl/19451947nueva-vida-para-reconvertirse-hombre

24. Buero L. Sentido del trabajo. Umbrales revista de actualidad religiosa latinoamericana, $\mathrm{N}^{\circ} 119$ [online] [acceso 17 Abril 2014] Disponible en: http://

www.umbrales.edu.uy/umbrales/rev129/15_22.h $\mathrm{tm}$

25. Rodríguez E. El sentido del sufrimiento, experiencias de los enfermos del cáncer. Ars Medica, revista de estudios médico humanísticos, vol.3 n³. [online] [acceso 28 Abril 2014] Disponible en: http://escuela.med.puc.cl/publ/arsmedica/arsme dica3/06_Rodriguez.html

26. Larrañaga, R. Conformidad con la muerte. Crítica Política [sitio web] [acceso 20 Abril 2014]. Disponible en: 